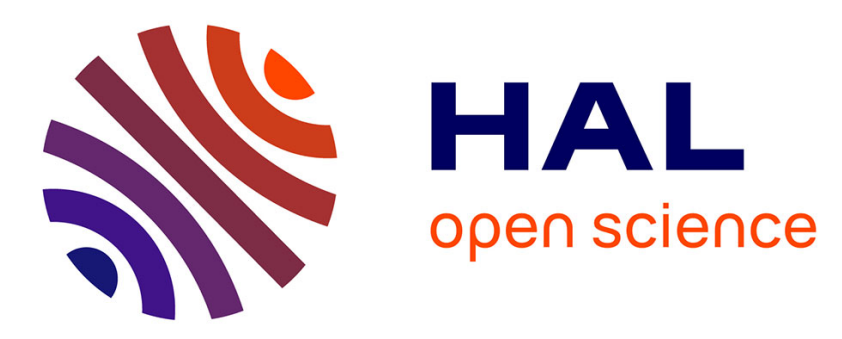

\title{
A Novel Statistical Model of OFDM Signals with Optimal Tone Reservation PAPR Reduction for EVM Theoretical Analysis
}

Mariam Elhassan, Matthieu Crussière, Jean-François Hélard, Youssef Nasser, Oussama Bazzi

\section{To cite this version:}

Mariam Elhassan, Matthieu Crussière, Jean-François Hélard, Youssef Nasser, Oussama Bazzi. A Novel Statistical Model of OFDM Signals with Optimal Tone Reservation PAPR Reduction for EVM Theoretical Analysis. IEEE International Symposium on Personal, Indoor and Mobile Radio Communications, Sep 2018, bologne, Italy. hal-01883178

\section{HAL Id: hal-01883178 https://hal.science/hal-01883178}

Submitted on 27 Sep 2018

HAL is a multi-disciplinary open access archive for the deposit and dissemination of scientific research documents, whether they are published or not. The documents may come from teaching and research institutions in France or abroad, or from public or private research centers.
L'archive ouverte pluridisciplinaire HAL, est destinée au dépôt et à la diffusion de documents scientifiques de niveau recherche, publiés ou non, émanant des établissements d'enseignement et de recherche français ou étrangers, des laboratoires publics ou privés. 


\title{
A Novel Statistical Model of OFDM Signals with Optimal Tone Reservation PAPR Reduction for EVM Theoretical Analysis
}

\author{
Mariam ElHassan*†, Matthieu Crussière*, Jean-François Hélard*, Youssef Nasser ${ }^{\ddagger}$, Oussama Bazzi ${ }^{\dagger}$ \\ * Univ Rennes, INSA Rennes, CNRS, IETR-UMR6164, F-35000 Rennes, France \\ $\dagger$ Lebanese University, Faculty of Sciences, Rafic Hariri University Campus, Hadath, Lebanon \\ $\ddagger$ American University of Beirut, Beirut, Lebanon 11072020 \\ Email: \{mariam.el-hassan, matthieu.crussiere, jean-francois.helard\}@ insa-rennes.fr, \\ yn10@aub.edu.lb, obazzi@ul.edu.lb
}

\begin{abstract}
In this paper, we study the statistical distribution of the OFDM time-domain signal amplitude to which the optimal tone reservation (TR) PAPR reduction solution is applied. This study comes as a major step towards the derivation of the analytical expression of the error vector magnitude (EVM) metric on OFDM waveforms when non-linear amplification is used. We demonstrate through simulations that this distribution has a bimodal behavior and can be tightly modeled as a superposition of two probability density functions whose expressions are given and whose parameters are obtained through proper estimation. From such analysis, we provide the complete distribution expression of the PAPRreduced OFDM signal, which can be further exploited for the derivation of performance bounds of the TR PAPR reduction strategy.
\end{abstract}

Index Terms-PAPR reduction, Tone Reservation, Clipping, EVM, Probability distribution

\section{INTRODUCTION}

Over the last decades, multi-carrier modulations and especially Orthogonal Frequency Division Multiplexing (OFDM) have been recognized as a key transmission scheme allowing for high transmission data rates over dispersive channels. However, a major problem of multi-carrier modulations remains the high peak to average power ratio (PAPR) of the generated timedomain signal, which translates into potentially strong power efficiency losses when amplifying the signal with High Power Amplifiers (HPA).

The power efficiency issue related to the amplification of multicarrier signals brings challenges to researchers which can essentially be categorized as follows. A first strategy is to linearize the HPA itself by means of predistortion techniques with the aim of benefiting from a larger linear zone. HPA linearization is today a quite mature technique and is already widely implemented. A second solution, which can be viewed as complementary to the former, is to process the baseband signal so as to reduce its dynamic range, i.e. reduce its PAPR. Since the introduction of the OFDM concept, numerous algorithms have been introduced for
PAPR reduction indeed. The simplest one is clipping [1] but it yields signal distortion, whereas advanced approaches such as active constellation extension [2] or tone reservation (TR) [3] can be used. TR offers a high potential gain, downward compatible method and was adopted by standards like Digital Video Broadcasting - Second Generation Terrestrial (DVB-T2) [4].

Despite a large number of available algorithms for PAPR reduction, analytical performance of these algorithms has not been enough studied. Moreover, the overall question of the efficiency of the PAPR reduction operation suffers a lack of theoretical (not algorithmic) analysis. Indeed, the analytical evaluation of the performance metrics such as error vector magnitude (EVM) and adjacent channel power ratio (ACPR) is of high interest to predict the expected power efficiency gains brought by a given PAPR reduction algorithm. Besides, such evaluation would allow to precisely determine the optimal parameters of the selected PAPR reduction algorithm and optimally set the operating point of the HPA.

In this context, a recent study aimed at evaluating the analytical performance of the clipping PAPR reduction method by analytical derivations of the EVM and ACPR [5]. Nowadays, none of the advanced PAPR reduction techniques such as TR has been analytically evaluated. In that perspective, we propose in this paper to model the statistical distribution of the amplitude of OFDM signals when a TR PAPR reduction scheme is activated. This work is an essential first step to further derive the analytical expression of EVM for instance. To envision the computation of performance bounds, our study focuses on the optimal TR PAPR reduction solution, called Quadratic Constraint Quadratic Problem (QCQP). In this paper, we investigate the statistical effect of the QCQP on the OFDM signal amplitude and propose an accurate statistical model for such signal amplitude, reflecting the modifications brought by the QCQP algorithm. Our main contribution, is to 
introduce, through an in-depth analysis, an analytical expression of the distribution of the amplitude of TRbased PAPR-reduced OFDM signals, and then derive the signal EVM. This will definitely open the door to tune the TR-QCQP PAPR solution.

The rest of this paper is organized as follows. In section II we describe the system model and detail the PAPR reduction block in the case of clipping and of TR with mathematical notations. We also detail the considered HPA model and define EVM for the case of clipping and tone reservation. In section III we summarize the steps taken in [5] in order to evaluate the EVM. We then move in section IV to TR technique and show how we evaluate signal distribution in this case. Section VI concludes this work.

\section{System ModeL}

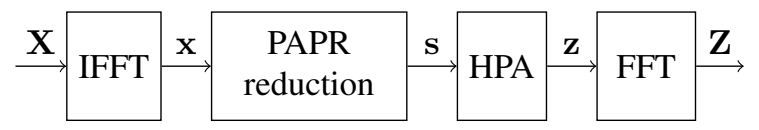

Fig. 1: System model

Let us consider an OFDM transmission chain as depicted in Fig. 1 where the time domain baseband signal is subject to PAPR reduction operation and then amplified by a non-linear HPA. In the sequel, the considered PAPR reduction methods will be clipping and TR. The DVB-T2 frequency domain frame structure [4] is considered with $N$ subcarriers along with the positions of the reserved tones in case of TR algorithm.

Let $\mathbf{X} \in \mathbb{C}^{N}$ be the original frequency domain symbol vector and $\mathbf{x} \in \mathbb{C}^{N}$ its corresponding time domain symbol vector. $\mathbf{s}$ is the so obtained symbol vector after peak reduction and $\mathbf{z}$ that obtained after high power amplification. $\mathbf{Z}$ is the corresponding frequency domain symbol vector representing the signal after demodulation at the receiver side. Without loss of generality, we will consider in the following an OFDM signal with $N=1024$ sub-carriers with 16-QAM constellation. Our aim is to derive EVM expression between the original and the transmitted signal, depending on the implemented PAPR reduction algorithm. In the following sections, we detail the PAPR reduction block model for both clipping and TR methods, the model used for the HPA and the general EVM definition.

\section{A. PAPR Reduction}

1) Clipping: After clipping, the obtained time domain signal is simply given by:

$$
\mathbf{s}(k)= \begin{cases}x(k) & \text { if }|x(k)| \leq V_{\text {clip }} \\ V_{\text {clip }} e^{j \angle x(k)} & \text { if }|x(k)|>V_{\text {clip }},\end{cases}
$$

where $s(k)$ (resp. $x(k)$ ) stands for the $k^{\text {th }}$ element of vector $\mathbf{s}$ (resp. $\mathbf{x}$ ). $V_{\text {clip }}$ is the clipping amplitude level and $\angle x(k)$ is the phase of sample $x(k)$.
2) Tone Reservation: Let us consider c the time domain peak cancellation signal added to $\mathbf{x}$ using the TR principle:

$$
\mathbf{s}=\mathbf{x}+\mathbf{c}
$$

Vector $\mathbf{c}$ is built upon the $R$ peak reduction tones (PRT) according to a subset of subcarriers $\beta$ as defined in the DVB-T2 specifications for instance. Let $\mathbf{C}$ be the vector containing the PRTs at positions $\beta$ and zeros elsewhere. $\mathbf{C}$ and $\mathbf{X}$ lie in disjoint frequency subspaces, i.e. $X_{k}=0, \forall k \in \beta$ and $C_{k}=0, \forall k \in \beta^{c}$, which ensures that TR is an in-band distortion-less method ${ }^{1}$. Basically, $\mathbf{C}$ is calculated from the optimization problem proposed by Tellado in [3] as:

$$
\begin{aligned}
& \min \tau \\
& \text { subject to }\|\mathbf{x}+\mathbf{Q C}\|_{\infty}^{2} \leqslant \tau
\end{aligned}
$$

where $\mathbf{Q}$ is the inverse fast Fourier transform (IFFT) matrix and $\|.\|_{\infty}$ denotes infinity norm. As some standards such as DVB-T2 add a power constraint to the maximum power of a PRT, the following condition holds:

$$
\|\mathbf{C}\|_{\infty}^{2} \leqslant P_{\max }+P_{x}
$$

where $P_{x}$ is the power of the input signal $\mathbf{x}$ and $P_{\max }$ is the maximum allowed power (in $d B$ ) above the signal power. The problem is thus a convex optimization problem of type quadratically constraint quadratic program (QCQP) [7]. The QCQP problem formulation is the optimal solution for TR and gives the upperbound of PAPR reduction level using TR. However, QCQP is complex and introduces high latency due to its computation time. To solve the QCQP problem, several sub-optimal methods have been proposed in the literature. This is usually done by linear operations on the kernel signal $\mathbf{c}$.

In this paper we consider the QCQP algorithm aiming to find the theoretical limits of EVM in the case of TR.

\section{B. Power Amplifier}

We consider the memoryless amplitude to amplitude characteristic of the HPA by the following polynomial model [8]:

$$
H_{P A}(r)=\sum_{l=0}^{L_{p}-1} b_{2 l+1} r^{2 l+1}
$$

where $L_{p}$ is the order of the HPA polynomial model and $b_{2 l+1}$ its odd indexed coefficients.

\section{EVM Expression}

EVM is a metric that measures the in-band distortion level of a signal. It is the amount of deviation of the constellation points of $\mathbf{Z}$ with respect to the original signal $\mathbf{X}$. Considering an $N$-point IFFT at nominal

\footnotetext{
${ }^{1}$ it is worth mentioning that the HPA includes however an in-band and out-of-band distortion
} 
sampling rate, EVM can be evaluated on the time domain samples. Let $\mathbf{s}_{\text {ref }}$ be the reference signal vector to which the final signal vector $\mathbf{z}$ is compared for EVM evaluation. We have then:

$$
E V M=\sqrt{\frac{\mathrm{E}\left\{\left|z(k)-s_{\text {ref }}(k)\right|^{2}\right\}}{\mathrm{E}\left\{\left|s_{\text {ref }}(k)\right|^{2}\right\}}}
$$

The denominator is simply equal to the reference signal power. Depending whether the PAPR reduction algorithm is distortion-less or not, $\mathbf{s}_{\text {ref }}$ will be taken as $\mathbf{x}$ or $\mathbf{s}$ in the following. Using the polar coordinates with amplitude $r$ and phase $\phi$ of each signal sample, the expectation in the numerator becomes:

$$
\int_{-\infty}^{\infty} \int_{0}^{2 \pi}\left|z(r, \phi)-s_{r e f}(r, \phi)\right|^{2} f_{r e f}(r, \phi) \mathrm{d} r \mathrm{~d} \phi
$$

where $f_{r e f}(r, \phi)$ is the probability density function (PDF) governing the random amplitude and phase of the reference signal samples. Then, since $\phi$ follows a uniform distribution over $[-\pi, \pi]$, the expectations can be simplified yielding the general EVM expression:

$$
E V M=\sqrt{\frac{\int_{-\infty}^{\infty}|z(r)-r|^{2} f_{r e f}(r) \mathrm{d} r}{\int_{-\infty}^{\infty} r^{2} f_{r e f}(r) \mathrm{d} r}}
$$

It follows that computing the EVM relies on the knowledge of the amplitude PDF in the reference signal $f_{r e f}(r)$. The level of distortion captured by the EVM measurement depends on the HPA non-linearity but can be introduced at the PAPR reduction level depending on the used technique. Indeed, if clipping is used, distortion is introduced to the signal whereas TR does not bring any data modification due to the use of orthogonal PRT.

Thus, in the following, when computing the EVM in the case of clipping, we will have to consider the signal $\mathbf{x}$ as the reference signal. In the case of TR, the reference signal will rather be chosen as $\mathbf{s}$ since distortions will only be produced by the HPA. In other words, for the former case we will have $f_{\text {ref }}(r)=$ $f_{x}(r)$, and $f_{r e f}(r)=f_{s}(r)$ for the latter.

\section{EVM EXPRESSION IN THE CASE OF CLIPPING}

In case of clipping, EVM has to be evaluated from (8) using the amplitude distribution of signal samples $x(k)$.

\section{A. Time Domain Amplitude Distribution}

Due to the central limit theorem, if the number of subcarriers $N$ is large, real and imaginary parts of the time domain OFDM signal can be approximated by a centered normal distribution. Thus, the amplitude of the time domain OFDM signal follows a Rayleigh distribution:

$$
f_{x}(r)=\frac{2 r}{P_{x}} e^{-\frac{r^{2}}{P_{x}}}, \quad r \geq 0
$$

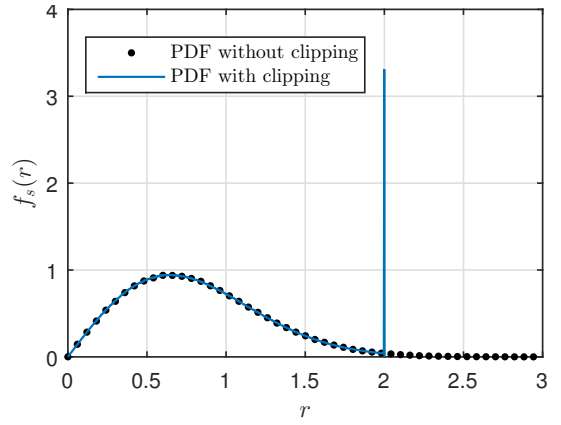

Fig. 2: PDF of the amplitude of the time domain signal with and without clipping at $V_{\text {clip }}=2$

and 0 elsewhere. The amplitude distribution after the clipping operation is easily obtained from the expression of $\mathrm{s}$ in (1) as:

$$
f_{s}(r)=\left\{\begin{array}{ll}
f_{x}(r) & \text { if } r<V_{\text {clip }} \\
P\left\{r \geq V_{\text {clip }}\right\} \delta\left(r-V_{\text {clip }}\right) & \text { if } r \geq V_{\text {clip }}
\end{array},\right.
$$

where $\delta(r)$ is the Dirac impulse and $P\left\{r \geq V_{\text {clip }}\right\}$ is the probability of $r$, the amplitude of $x(k)$ to be higher than $V_{\text {clip }}$ :

$$
P\left\{r \geq V_{\text {clip }}\right\}=\int_{V_{\text {clip }}}^{\infty} f_{x}(r) \mathrm{d} r=e^{\frac{-V_{\text {clip }}^{2}}{P_{x}}}-1
$$

Both PDFs, with and without clipping, are depicted in Fig. 2. It is observed that $f_{s}(r)$ contains a strong peak instead of a long tail as is the case with $f_{x}(r)$.

\section{B. EVM computation}

To derive the EVM computation from (8), it is clear to consider the output signal $z(r)$, accounting for the HPA and clipping effects. The sample $z(r)$ is the amplified version of $s(r)$, this latter being the clipped version of $x(r)$. Hence, we have:

$$
z(r)= \begin{cases}H_{P A}(x(r)) & \text { if } r<V_{\text {clip }} \\ H_{P A}\left(V_{\text {clip }}\right) & \text { if } r \geq V_{\text {clip }}\end{cases}
$$

The expectation in (8) can thus be expressed as:

$\int_{0}^{V_{\text {clip }}}\left|r-H_{P A}(r)\right|^{2} f_{x}(r) \mathrm{d} r+\int_{V_{\text {clip }}}^{\infty}\left|r-H_{P A}\left(V_{\text {clip }}\right)\right|^{2} f_{x}(r) \mathrm{d} r$

Using (5), it is then possible to lead the integral computation and obtain the EVM as [5]:

$$
\begin{aligned}
& {\left[1+\sum_{l=0}^{L_{p}-1} \sum_{l^{\prime}=0}^{L_{p}-1} v_{l l^{\prime}}\left(\gamma\left(l+l^{\prime}+2, \Lambda\right)+\Lambda^{l+l^{\prime}+1} e^{-\Lambda}\right)\right.} \\
& \left.\quad-u_{l}\left(\gamma(l+2, \Lambda)+\Lambda^{l+\frac{1}{2}} \Gamma\left(\frac{3}{2}, \Lambda\right)\right)\right]^{\frac{1}{2}}
\end{aligned}
$$

with $u_{l}=2 P_{x}^{l} \mathfrak{R}\left\{b_{2 l+1}\right\}$ and $v_{l l^{\prime}}=b_{2 l+1} b_{2 l^{\prime}+1}^{*} P_{x}^{l+l^{\prime}}$, $\Lambda$ the clipping ratio $V_{c l i p} / P_{x}$, and where $\gamma(x, s)$ and $\Gamma(x, s)$ stand for the lower and upper incomplete gamma functions, respectively. 


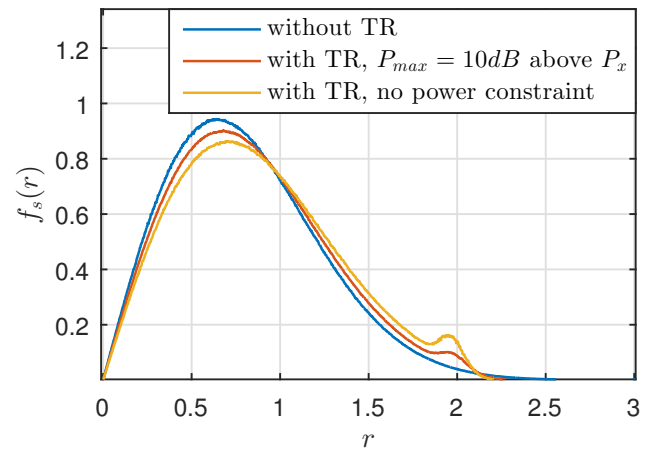

Fig. 3: Simulated PDF of the amplitude of the Time domain signal without TR, with TR without constraint, and with TR with $P_{\max }=10 \mathrm{~dB}$ constraint

\section{EVM EXPRESSION WITH QCQP-TR ALGORITHM}

As already explained, in case of TR algorithm, EVM is expressed from (8) but now substituting $f_{r e f}(r)$ with $f_{s}(r)$. A major step is hence to evaluate the distribution of the signal amplitude $\mathbf{s}(r)$ after TR. However, the amplitude of the time domain signal after TR is not as simple as in the case of clipping. Indeed, note that $x(k)$ and $c(k)$ follow a Gaussian complex distribution due to central limit theorem whereas central limit theorem can't be applied to $s(k)$ due to the dependence between $x(k)$ and $c(k)$ brought by the QCQP computation.

A thorough statistical analysis has to be led to attempt to properly model $f_{s}(r)$. As a first step, the empirical PDF of the amplitude of the simulated time domain signal after applying TR is shown in Fig. 3. The different empirical PDFs are plotted for the cases without power constraints and with a power constraint of $P_{\max }=10 \mathrm{~dB}$ on the PRT. It turns out that the amplitude of this signal follows a bimodal distribution, depending on the power constraint $P_{\max }$, with a main mode in the low signal amplitudes and a second mode in the high values. In the sequel, we aim at analyzing such bimodal distribution and deriving a model in terms of $P_{\max }$.

\section{A. Single Symbol Distribution}

To understand the origin of the second mode in the distribution, let us represent the amplitude distribution for one OFDM symbol, as proposed in Fig. 4. The bottom figure gives the amplitude of a single symbol after TR algorithm, and the upper figure depicts the corresponding amplitude histogram. It turns out that each symbol is composed of two subsets of samples. The first is the set of samples at the maximum amplitude value $\|\mathbf{s}\|_{\infty}=A_{\max }$ forming a peak in the histogram at $A_{\max }$ and the second is the set of the samples at a value less than $A_{\max }$ corresponding to the rest of the distribution. Differently from the clipping approach, for each symbol the value of the maximum
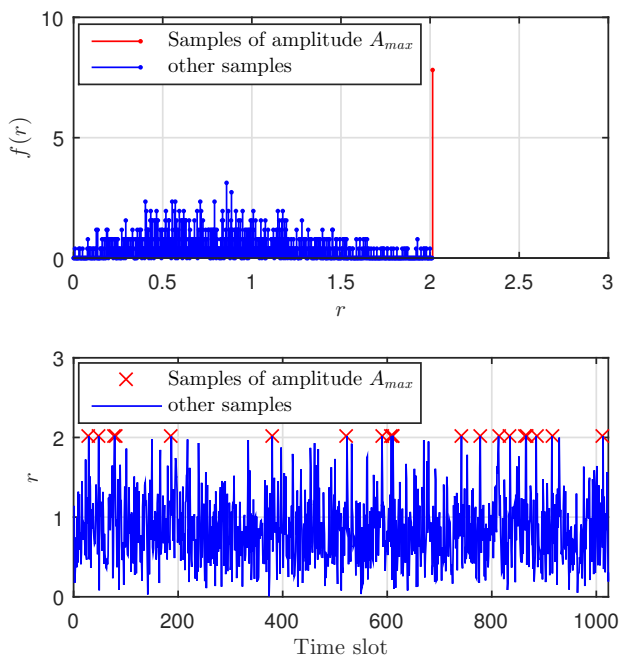

Fig. 4: Time domain representation of the amplitude of one symbol after optimal TR using QCQP solution, and related histogram.

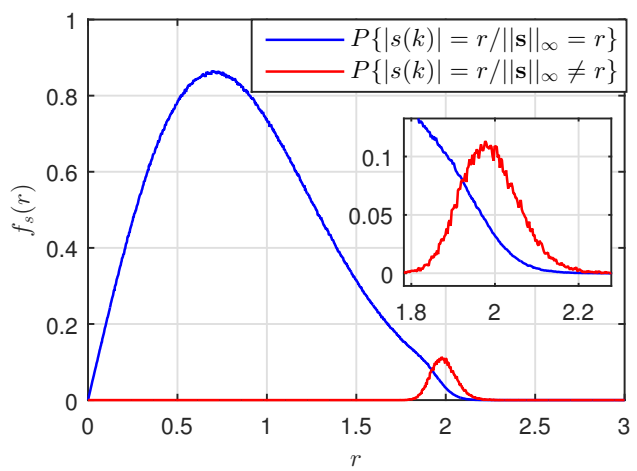

Fig. 5: Empirical distributions for $f_{s 1}(r)$ and $f_{s 2}(r)$ separately, QCQP applied (no power constraint)

$A_{\max }$ is different depending on the solution of the QCQP program to converge to the optimal minimum $\|\mathbf{s}\|_{\infty}$. Also, note that the observed distribution does not correspond to an actual clipping of the symbol amplitude; in fact, TR modifies the whole signal distribution and each sample can take a new value.

\section{B. Separated Distributions}

From the above observations, let us pursue the analysis by separating the distributions of the two described parts of each symbol. Let us model the bimodal distribution as a superposition of two different, though not independent, PDFs, that is

$$
\begin{gathered}
f_{s}(r)=(1-p) f_{s 1}(r)+p f_{s 2}(r) \\
\text { where }\left\{\begin{array}{l}
f_{s 1}(r)=\mathrm{P}\left\{\left|s_{k}\right|=r \mid r \neq\|\mathbf{s}\|_{\infty}\right\} \\
f_{s 2}(r)=\mathrm{P}\left\{\left|s_{k}\right|=r \mid r=\|\mathbf{s}\|_{\infty}\right\}
\end{array}\right.
\end{gathered}
$$

where $p$ is the expected value of the ratio between the number of samples at $A_{\max }$ and the total number of samples. Fig. 5 shows an example of the obtained 
amplitude distribution for the two parts that form $f_{s}(r)$. Hereafter, we are interested in giving an expression to each of these PDFs.

\section{Model of the distribution $f_{s 2}(r)$}

From the observed shape of the simulated $f_{s 2}(r)$ for different values of $P_{\max }$, a convenient model for $f_{s 2}(r)$ can be found by a generalized extreme value (GEV) distribution of parameters $\mu_{2}, \sigma_{2}$ and $k_{2}$ :

$$
f_{s 2}(r)=f_{G E V}\left(r ; \mu_{2}, \sigma_{2}, k_{2}\right)
$$

where,

$$
\begin{array}{r}
f_{G E V}(r ; \mu, \sigma, k)= \begin{cases}\frac{1}{\sigma} \mathrm{t}(r)^{k+1} e^{-\mathrm{t}(r)} & \text { if } r \in D \\
0 & \text { elsewhere }\end{cases} \\
\text { with } \mathrm{t}(r)= \begin{cases}\left(1+k\left(\frac{r-\mu}{\sigma}\right)\right)^{-1 / k} & \text { if } k \neq 0 \\
e^{-(x-\mu) / \sigma} & \text { if } k=0\end{cases}
\end{array}
$$

where $D$ is the support of the GEV distribution defined as:

$$
D= \begin{cases}{[\mu-\sigma / k ;+\infty)} & \text { if } k>0 \\ (-\infty ;+\infty) & \text { if } k=0 \\ (-\infty ; \mu-\sigma / k] & \text { if } k<0\end{cases}
$$

and $\mu, \sigma$ and $k$ are the location, scale and shape parameters of the distribution, respectively. The GEV distribution has been selected as it is the typical distribution that models the maximum of i.i.d sequences. We computed these parameters for each value of $P_{\max }$ using the probability weighted moment (PWM) estimation method [9], [10]. Fig. 6 shows the obtained variations of the GEV parameters in function of $P_{\max }$, along with an example of the GEV fitting $f_{s 2}(r)$ with and without power constraint. These curves will be taken as reference for the parameter setting of our model.

\section{Model of the distribution $f_{s 1}(r)$}

Coming back to the observation of Fig. 4, we can understand that for a particular maximum value $A_{\max }=\|\mathbf{s}\|_{\infty}$ the distribution of the samples in one OFDM symbol follows a Rayleigh distribution that is truncated (not clipped) at $A_{\max }$. Hence, it is convenient to introduce the following conditional PDF:

$$
f_{s 1}\left(r \mid A_{\max }\right)=f_{R a y}\left(r ; \sigma_{r}\right)\left(1-\mathrm{u}\left(r-A_{\max }\right)\right)
$$

where $f_{R a y}\left(r ; \sigma_{r}\right)$ denotes the Rayleigh PDF of parameter $\sigma_{r}$ and $\mathrm{u}(r)$ is the Heaviside unit step function. Note that the Rayleigh distribution is truncated for each symbol at an eventually different amplitude $A_{\max }$. Thus, a marginal PDF is obtained as:

$$
f_{s 1}(r)=\int_{-\infty}^{\infty} f_{s 1}\left(r \mid A_{\max }\right) f_{A_{\max }}(A) \mathrm{d} A
$$

$f_{A_{\text {max }}}(A)$ is the PDF of the maximum amplitude of each time-domain symbol:

$$
f_{A_{\max }}(A)=P\left\{\|\mathbf{s}\|_{\infty}=A\right\} .
$$
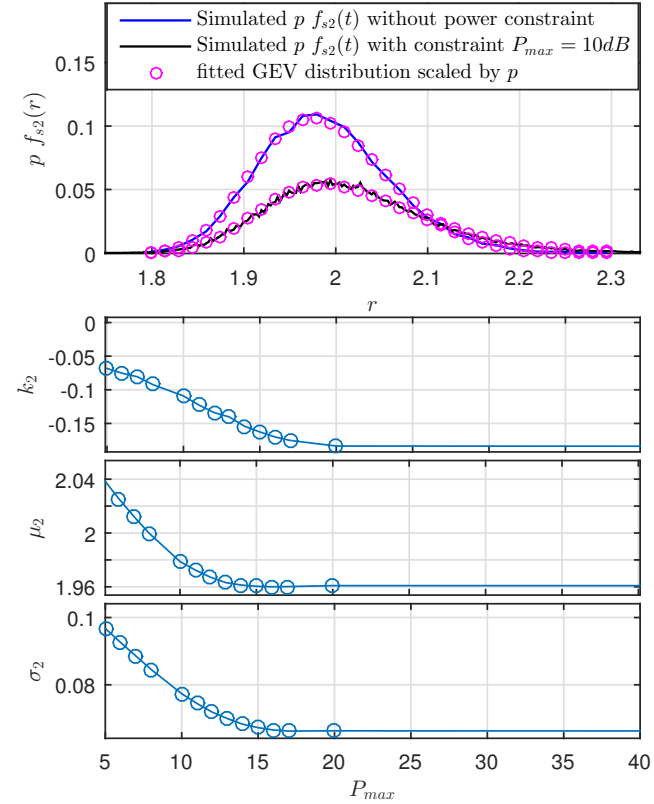

Fig. 6: Comparison between the empirical and GEVbased PDFs for $f_{s 2}(r)$. Skewness, position and scale parameters of the GEV distribution fitted to $f_{s 2}(r)$ in function of $P_{\max }$

Then, integrating (15) in (16) leads to:

$$
\begin{aligned}
& f_{s 1}(r)=f_{\text {Ray }}\left(r ; \sigma_{r 1}\right) \times \\
& \left(1-\int_{-\infty}^{\infty} \mathrm{u}(r-A) f_{A_{\max }}(A) \mathrm{d} A\right)
\end{aligned}
$$

The latter integral can further be derived as:

$$
\int_{-\infty}^{r} f_{A_{\max }}(A) \mathrm{d} A
$$

which can be identified as the Cumulative Density Function (CDF) of random variable $A_{\max }$. We thus finally obtain:

$$
f_{s 1}(r)=q f_{R a y}\left(r ; \sigma_{r 1}\right)\left(1-F_{A_{\max }}(r)\right)
$$

where $q$ is a normalization factor that ensures $\int f_{s 1}(r) d r=1$. Accordingly, the parameter $\sigma_{r 1}$ has to be found for PDF $f_{R a y}\left(r ; \sigma_{r 1}\right)$ and $f_{A_{\max }}(r)$ should be modeled in terms of $P_{\max }$. Fig. 7 shows an example of $f_{A_{\max }}(r)$ with and without the power constraint of $P_{\max }=10$. As shown in this figure, and with a similar study of $f_{s 2}(r), f_{A_{\max }}(r)$ can be modeled by a GEV distribution with parameters $\mu_{1}, \sigma_{1}$ and $k_{1}$ estimated from the PWM method. Finally, the form of $f_{s 1}(r)$ and the corresponding Rayleigh fitting can easily be found as presented in Fig. 8. Eventually, the values of $\sigma_{r_{1}}, \mu_{1}, \sigma_{1}, k_{1}$ can be used as reference in the global statistical model. 
$E V M=\sqrt{\frac{\int_{-\infty}^{\infty}\left|r-\sum_{l=0}^{L_{p}-1} b_{2 l+1} r^{2 l+1}\right|^{2}\left((1-p) q f_{R a y}\left(r ; \sigma_{r_{1}}\right)\left(1-F_{G E V}\left(r ; \mu_{1}, \sigma_{1}, k_{1}\right)\right)+p f_{G E V}\left(r ; \mu_{2}, \sigma_{2}, k_{2}\right)\right) \mathrm{d} r}{\int_{-\infty}^{\infty}|r|^{2}\left((1-p) q f_{R a y}\left(r ; \sigma_{r_{1}}\right)\left(1-F_{G E V}\left(r ; \mu_{1}, \sigma_{1}, k_{1}\right)\right)+p f_{G E V}\left(r ; \mu_{2}, \sigma_{2}, k_{2}\right)\right) \mathrm{d} r}}$
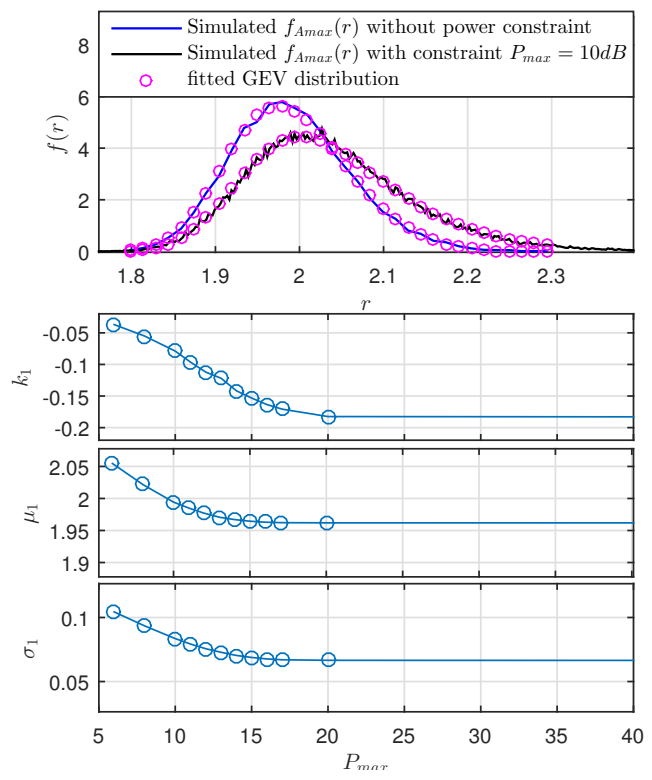

Fig. 7: Comparison between the empirical and GEVbased PDFs for $f_{A_{\max }}(r)$. Parameters of the GEV distribution fitted to $f_{A_{\max }}(r)$ in terms of $P_{\max }$
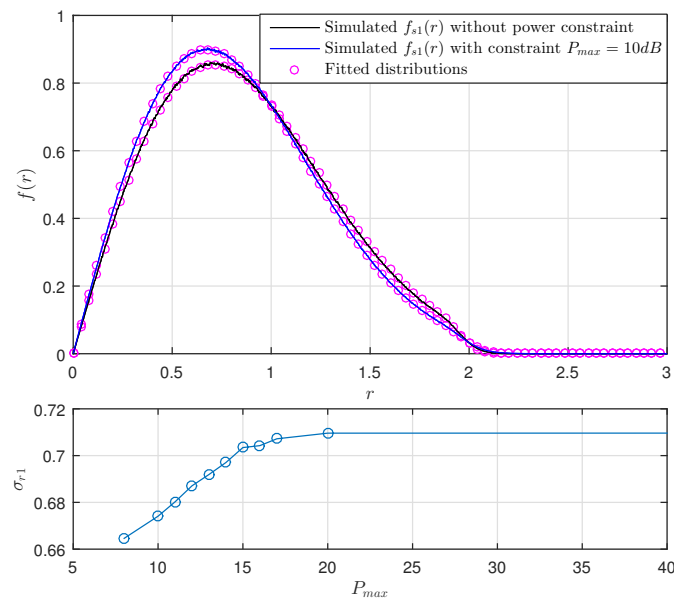

Fig. 8: Comparison between the empirical and modified Rayleigh-based PDFs for $f_{s 1}(r)$. Parameter of the fitted Rayleigh distribution of $f_{s 1}(r)$ in function of $P_{\max }$.

\section{E. EVM expression for the TR method}

From the so obtained model of $f_{s}(r)$ as a bimodal distribution composed of a GEV and a modified Rayleigh distribution, we obtain the EVM expression given on top of the page.

\section{CONCLUSION}

In this paper, we have led a thorough analysis on the amplitude distribution of OFDM signals with PAPR reduction based on clipping and TR. We have provided the statistical model for each of the cases allowing for deriving the EVM of the signal at the output of the non-linear HPA. A major contribution of the paper has been to introduce and propose a novel distribution to model the amplitude of the OFDM signal using the optimal QCQP solution for TR PAPR reduction. The proposed distribution has been shown to tightly model the statistical behavior of the signal. This strong result represents an essential first step for further analytical derivation of the EVM of OFDM signals with PAPR reduction. In particular, the provided model is expected to allow a derivation of the TR-based PAPR reduction methods performance.

\section{ACKNOWLEDGEMENT}

The authors would like to thank the european Green Tea project for their support of this work.

\section{REFERENCES}

[1] R. Yoshizawa and H. Ochiai, Effect of Clipping and Filtering with Distortionless PAPR Reduction for OFDM Systems, in 2015 IEEE 82nd Vehicular Technology Conference (VTC2015Fall), 2015, pp. 15.

[2] B. S. Krongold and D. L. Jones, PAR reduction in OFDM via active constellation extension, IEEE Trans. Broadcast., vol. 49, no. 3, pp. 258268, Sep. 2003.

[3] J. Tellado and J. M. Cioffi, Peak power reduction for multicarrier transmission, in IEEE GLOBECOM, 1998, vol. 99, pp. 59.

[4] ETSI. Frame structure channel coding and modulation for a second generation digital terrestrial television broadcasting system (DVB-T2). EN 30255 v1.4.1, European Telecommunications Standards Institute (ETSI), 2015.

[5] A. Cheaito, M. Crussière, J. F. Hélard, and Y. Louet, Quantifying the Memory Effects of Power Amplifiers: EVM ClosedForm Derivations of Multicarrier Signals, IEEE Wire. Commun. Letters, vol. 6, no. 1, pp. 3437, Feb. 2017.

[6] M. Issa, A-K Ajami, H. Artail, Y. Nasser, 'An approximation for the distribution of the peak-to-average power ratio in carrier-aggregated OFDM signals using level crossing rate analysis', pp. 1-8, in the proceedings of WiMob 2017.

[7] J. Tellado and J. M. Cioffi, PAR Reduction in Multicarrier Transmission Systems, ANSI Document, T1E1.4 Technical Subcommittee, vol. 4, pp. 97367, 1998.

[8] J. Kim and K. Konstantinou, Digital predistortion of wideband signals based on power amplifier model with memory, Electronics Letters, vol. 37, no. 23, pp. 14171418, Nov. 2001.

[9] P. Ribereau, E. Masiello, and P. Naveau, Skew generalized extreme value distribution: Probability-weighted moments estimation and application to block maxima procedure, Communications in Statistics - Theory and Methods, vol. 45, no. 17, pp. 50375052, Sep. 2016.

[10] J. R. M. Hosking, J. R. Wallis, and E. F. Wood, Estimation of the Generalized Extreme-Value Distribution by the Method of Probability-Weighted Moments, Technometrics, vol. 27, no. 3, p. 251, Aug. 1985. 